\title{
Primary pulmonary adenocarcinoma in roe deer - a case report
}

\author{
MICHAŁ BEDNARSKI, MARCJANNA WIMONĆ*, RAFAŁ KOLENDA*, \\ EDMUND WLEKLIŃSKI**, DAWID KRÓL***, MAREK HOUSZKA****
}

Department of Epizootiology and Clinic of Birds and Exotic Animals, Faculty of Veterinary Medicine, University of Environmental and Life Sciences, pl. Grunwaldzki 46, 50-366 Wrocław, Poland

*Department of Biochemistry and Molecular Biology, Faculty of Veterinary Medicine, University of Environmental and Life Sciences, ul. C. K. Norwida 31, 50-375 Wrocław, Poland

**Forest District Piaski, ul. Drzęczewska 1, 63-820 Piaski, Poland

${ }^{* * * D}$ Department of Immunology, Pathophysiology and Veterinary Preventive Medicine, Faculty of Veterinary Medicine, University of Environmental and Life Sciences, ul. C. K. Norwida 31, 50-375 Wrocław, Poland

****Department of Pathology, Faculty of Veterinary Medicine, University of Environmental and Life Sciences, ul. C. K. Norwida 31, 50-375 Wrocław, Poland

Bednarski M., Wimonć M., Kolenda R., Wlekliński E., Król D., Houszka M. Primary pulmonary adenocarcinoma in roe deer - a case report

\section{Summary}

Lung adenocarcinoma develops after the neoplastic transformation of pneumocytes or bronchial epithelium. It is chronic and slowly progressive in nature and the clinical signs are only seen in cases that have developed tumours of considerable size. There has been no report of lung adenocarcinoma in game animals. This case study reports a lung adenocarcinoma in a 4-year-old dead roe deer (Capreolus capreolus) from the Forest District Piaski, Greater Poland Province of Poland. During necropsy, a large mass measuring $15 \times 20 \mathrm{~cm}$ was found in the caudal lobe of the right lung. The tumour was of grey or pinkish-grey colour, solid texture and had a clear delineation between the affected and non-affected areas. In the remaining lobes, numerous small nodules of 2-20 mm in diameter with a similar structure were found. A great deal of mucoid and mucopurulent fluid throughout the bronchial tree was observed. The thoracic lymph nodes were found enlarged. The histological analysis identified the large mass found as bronchoalveolar papillary lung adenocarcinoma. The PCR for JSRV was performed and the result was negative. To the best of our knowledge, this is the first report of the lung adenocarcinoma in a roe deer.

Keywords: lung adenocarcinoma, roe deer, lung cancer, game animals

Lung adenocarcinoma develops after the neoplastic transformation of pneumocytes or bronchial epithelium. The World Health Organization (WHO) has classified lung adenocarcinoma as a malignant epithelial tumour with glandular differentiation, showing various growth patterns: i.e. acinar, papillary, bronchioloalveolar, solid with mucin production or a mixture of these patterns (17). The disease is chronic, slowly progressive and the clinical signs are seen only in cases that have developed tumours of considerable size (1). Affected animals may show progressive emaciation, weight loss and respiratory compromise. The tumour growth can be accompanied by overproduction of fluid in the lungs, which further compromises the normal respiration $(2,14)$. Macroscopic examination shows grey, solid tumours or infiltrations clearly separated from normal pulmonary tissue. According to its location, lung adenocarcinoma can be classified as bronchial, bronchioalveolar or alveolar type (17).
The factors causing pulmonary adenocarcinomas in animals can be divided into two groups: non-infectious and infectious. The former is non-transmissible and its incidence in animals is low, while the latter is a transmissible lung tumor known as ovine pulmonary adenocarcinoma (OPA) or Jaagsiekte $(5,14,20)$. The etiology of OPA is associated with Jaagsiekte Sheep Retrovirus (JSRV) infection, as this is the only known virus that causes lung adenocarcinoma. This cancer progresses slowly and is economically significant in South Africa, Scotland, and Peru (3). The virus has been reported as capable of being transmitted among animals through close contact (6). The JSRV is found worldwide (with the exception of Australia and New Zealand) and mainly affects domesticated sheep, but can also affect other species, like Sardinian moufflon (Ovis musimon) and very rarely goats $(13,14)$. The JSRV infection leads to the transformation of various lung epithelial cells, such as 


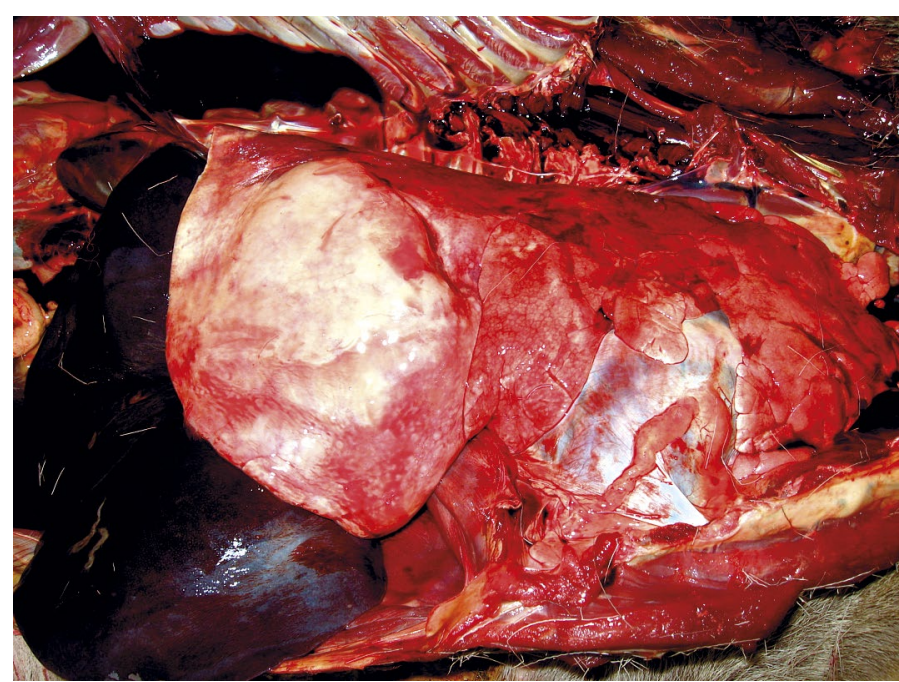

Fig. 1. Massive tumour in the caudal lobe of the right lung $(15 \times 20$ centimeters $)$

type II pneumocytes in the pulmonary alveoli and Clara cells in the bronchi.

In game animals there has been no report about primary lung adenocarcinoma. Therefore, it is important to report this case of pulmonary adenocarcinoma in roe deer (Capreolus capreolus).

\section{Case report}

In February 2012, a 4-year-old dead roe (Capreolus capreolus), originally from the Forest District Piaski, Greater Poland Province, Poland, was brought to the Department of Epizootiology with a Clinic of Birds and Exotic Animals, in Wroclaw. According to the observations of the forest inspectorate employees, the animal came from a herd of 12-15 animals and died suddenly. Shortly after death, it was transported to the Department of Epizootiology for necropsy. External examination of the animal revealed no skin lesions but the presence of an ecto-parasite, Lipoptena cervi, was observed. The general body hydration and nutritional status of the animal was bad. Internal post-mortem examination further confirmed that the animal was in poor health. During examination of the thoracic cavity, a large mass measuring $15 \times 20 \mathrm{~cm}$ was found in the caudal lobe of the right lung (Fig. 1). The tumour was of grey and pinkish-grey colour with a solid texture and had a clear delineation between the affected and non-affected areas (Fig. 2). In the remaining lobes, a number of small nodules ranging from $2-20 \mathrm{~mm}$ in diameter with a similar texture were observed. A great deal of mucoid and muco-purulent fluid was found during the necropsy throughout the bronchial tree. A considerable increase in the size of the thoracic lymph nodes was also noted. There were no other changes found in the rest of the respiratory system or in any other organ system during macroscopic examination of the cadaver. For the histopathological examination, a few small samples of the mass found in the right lung were fixed in formalin solution. Paraffin sections were prepared and stained with haematoxylin/eosin. In the microscopic examination, mass sections depicted focal points of proliferation of cuboidal or low columnar epithelial cells forming alveolar structures with papillary invaginations into their lumen (Fig. 3). The proliferation of epithelium was accompanied by spilled lympho- and histiocytic inflammatory infiltrations. In conclusion, the histological analysis revealed

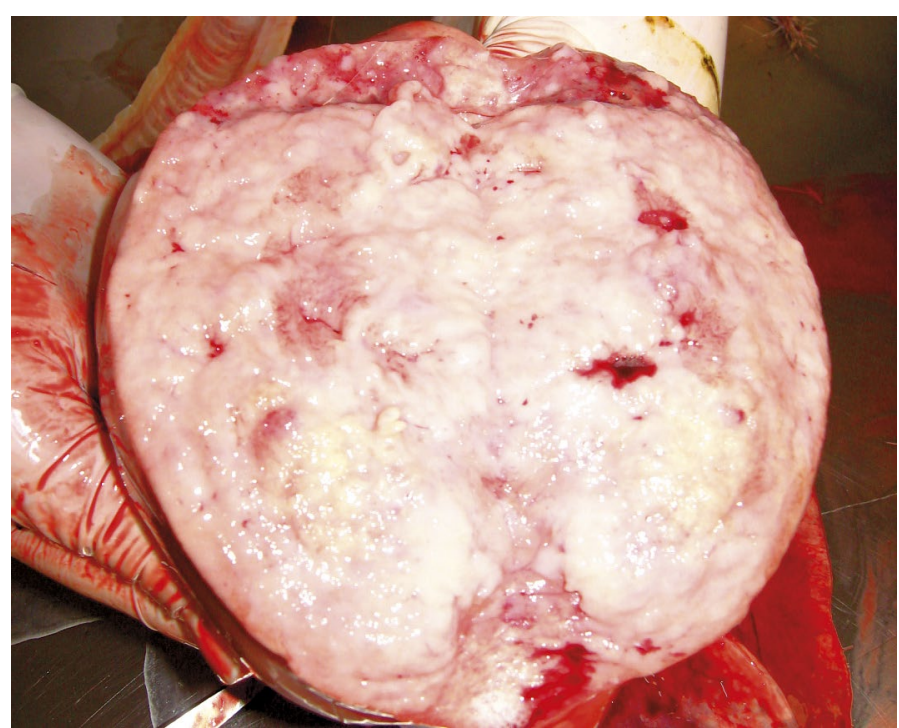

Fig. 2. Cross section through the tumour

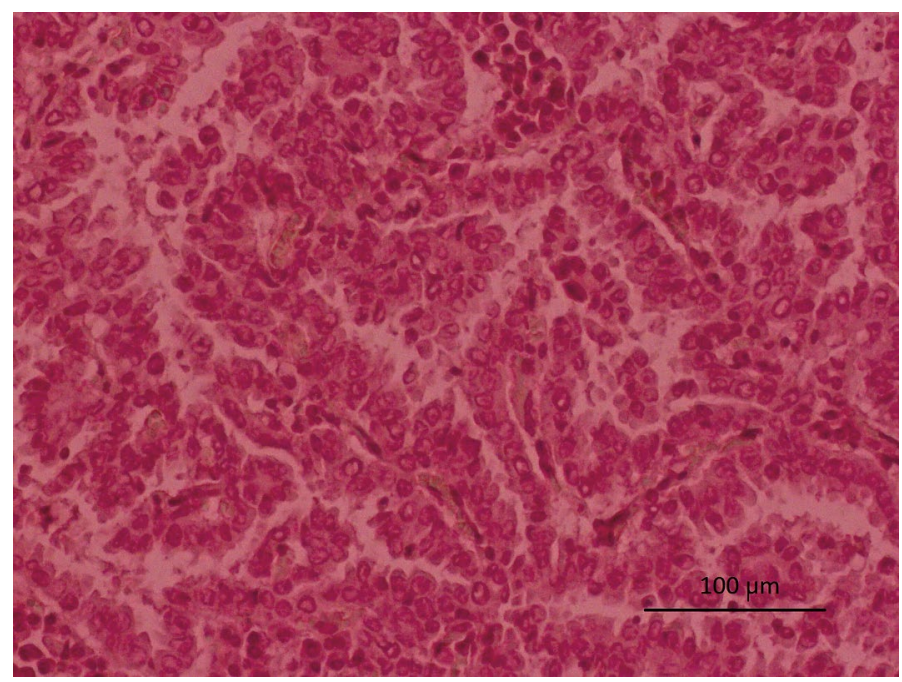

Fig. 3. Bronchoalveolar lung adenocarcinoma: proliferation of cuboidal or low columnar epithelial cells forming alveolar structures with papillary invaginations into their lumen (H-E staining)

that the large mass found in the lung was a bronchoalveolar papillary lung adenocarcinoma. There were no metastases in thoracic lymph nodes.

Additionally, PCR for JRSV was performed. A sample of tumor tissue was stored at $-80^{\circ} \mathrm{C}$ until further investigation. DNA extraction was performed with Syngen DNA Mini Kit (Syngen Biotech, Poland) according to manufacturer's instruction. A single step JSRV-specyfic PCR was performed using the method described by Palmarini et al. (11) and Kycko at al. (6). The PCR results was negative.

\section{Discussion}

Primary lung cancer in domesticated animals is rarely reported. What is more, there are no reports about primary lung cancer in game animals (18). In this study for the first time we macroscopically and microscopically describe lung adenocarcinoma in roe deer.

Lung adenomatosis in domesticated animals is rarely reported and its incidence in pet animals is low, but there are known cases in dogs and cats (8). This type of cancer is most commonly diagnosed in cats and dogs over 10 
years of age. Secondary metastases to the lymph nodes and the heart are most common (12). The study of Lucena et al., (7) reported four cases of lung adenocarcinoma in cattle out of 6706 animals tested. There is another report of lung adenocarcinoma in an adult cow but no other information about lung adenocarcinoma in cattle is available in literature (16). Lung cancer in ruminants is rare with the exception of OPA. The incidence of the OPA, as a transmissible disease, in sheep can be high and its typical prevalence ranges from $2 \%$ to $5 \%$. In an affected flock, the disease can be responsible for $2-5 \%$ of the annual mortality, although losses up to $20-50 \%$ have been reported. The highest morbidity and mortality rates were observed in a flock in the first few years after initial diagnosis of OPA and can be as high as $80 \%(13-15,19)$.

Sousa et al. (16) reported a case of lung tumour in a cow examined at a slaughterhouse. The tumour measured $10 \times 8 \mathrm{~cm}$, which is smaller than the one we found in the roe deer $(15 \times 20 \mathrm{~cm})$. Even smaller, multiple tumours about $3 \mathrm{~cm}$ in diameter were identified in the case of adenocarcinoma in $\operatorname{dog}(4)$. Tumours can vary in sheep from small nodules to solid masses. Sonawane et al. (15) described variable-sized nodules on the surface of lungs ranging from 1.5 to $3 \mathrm{~cm}$ in two sheep (13). In atypical cases of OPA, solitary or aggregated hard, white nodules with a dry cut surface have also been observed (2).

The localization of lung cancer in domesticated animals can vary. The tumour in the roe deer in our study was localized in the caudal lobe of the right lung, whereas in a case reported by Nakanishi et al., (9) the nodules and thickening of the pleura were found in the left lobe of the lung in a cat. The aforementioned study by Kim et al. (4) describes multiple pulmonary tumours localized in both lungs in the case of a 10-year-old female Yorkshire terrier. Another study reported a goat with multiple tumours localized in both lungs in the form of small grey nodules (10). In cases of OPA, tumours were found mainly in the apical, cardiac and ventral portions of the diaphragmatic lobes $(2,15)$.

The microscopic pictures of different cases of adenocarcinoma in animals are similar while we observed the centres of proliferation of epithelial cells forming alveolar structures with papillary projections into their light. The proliferation of epithelium was accompanied by more or less spilled inflammatory infiltrations. The microscopic structures found in roe deer tumours were similar to the previously reported cases of OPA $(2,15)$. The histological picture in the case of adenocarcinoma in a goat showed rectangular and column cells. A large number of neutrophils indicates an inflammatory reaction (10). Microscopic examination of samples from a cow showed bubble formation by neoplastic cells (16). In the case of a cat with lung adenocarcinoma, the microscopic image showed neoplastic lesions with a glandular structure and alveolar lesions with column cells (9). In contrast to our case, in ovine pulmonary adenomatosis the infiltrations of numerous macrophages were reported. Such infiltrations were observed in both natural and experimentally induced OPA. The microscopic examination of some samples from OPA revealed infiltration of neutrophils which may reflect a secondary bacterial infection $(2,15)$. Similar neutrophils infiltrations were also observed in our studied case of lung adenocarcinoma in the roe deer.

We hereby report the first case of bronchoalveolar papillary lung adenocarcinoma in the roe deer population. Although the etiological agent of this neoplasm is not yet identified, but taking into consideration the presence of endogenous retroviruses in genomes of deer, the infectious aetiology in this case cannot be excluded. However, the result of PCR shows that JSRV infections as an etiological factor of this neoplastic transformation was excluded. Analysis of more case reports of roe deer lung adenocarcinoma can contribute to a better understanding of the spread of the disease among wild animals.

\section{References}

1. Dungworth D. L., Hauser B., Hahn F. F., Wilson D. W., Haenichen T., Harkema $J$. R.: Histological classification of tumors of the respiratory system of domestic animals. Second series. VI. WHO, Armed Forces Institute of Pathology, Washington 1999.

2. Griffiths D. J., Martineau H. M., Cousens C.: Pathology and pathogenesis of ovine pulmonary adenocarcinoma. J. Comp. Pathol. 2010, 142, 260-283, doi: 10.1016/j.jcpa.2009.12.013.

3. Hecht S. J., Stedman K. E., Carlson J. O., DeMartini J. C.: Distribution of endogenous type B and type D sheep retrovirus sequences in ungulates and other mammals. Proc. Natl. Acad. Sci. USA 1996, 93, 3297-3302.

4. Kim J., Kwon S. Y., Cena R., Park S., Oh J., Oui H., Cho K. O., Min J. J., Choi J.: $\mathrm{CT}$ and PET-CT of a dog with multiple pulmonary adenocarcinoma. J. Vet. Med. Sci. 2014, 76, 615-620.

5. Kopczewski A., Twardowski H., Wardzinski S., Chyliński G.: The course of lung adenomatosis in the sheep. Med. Weter. 1978, 6, 347-349.

6. Kycko A., Jasik A., Reichert M.: Detection of Jaagsiekte sheep retrovirus in respiratory tract fluid and lung tissue of experimentally infected lambs. Bull. Vet. Inst. Pulawy 2008, 52, 9-13.

7. Lucena R. B., Rissi D. R., Kommers G. D., Pierezan F., Oliveira-Filho J. C., Macêdo J. T. S. A., Flores M. M., Barros C. S. L.: A retrospective study of 586 tumours in Brazilian cattle. J. Comp. Pathol. 2011, 145, 20-24, doi: 10.1016/ j.jcpa.2010.11.002.

8. Moulton J. E.: Tumors in Domestic Animals. $5^{\text {th }}$ ed. Wiley-Blackwell, Ames 2017.

9. Nakanishi M., Kuwamura M., Ueno M., Yasuda K., Yamate J., Shimada T. Pulmonary adenocarcinoma with osteoblastic bone metastases in a cat J. Small Anim. Pract. 2003, 44, 464-466.

10. Ortín A., Benito A. A., Lacasta D., Ferrer L. M., De Las Heras M.: Bronchioloalveolar carcinoma not related to jaagsiekte sheep retrovirus in a goat. Vet. Pathol. 2007, 44, 710-712, doi: 10.1354/vp.44-5-710.

11. Palmarini M., Cousens C., Dalziel R. G., Bai J., Stedman K., Demartini J. C., Sharp J. M.: The exogenous form of Jaagsiekte retrovirus (JSRV) is specifically associated with a contagious lung cancer of sheep. J. Virol. 1996, 70, 1618-1623.

12. Sato T., Ito J., Shibuya H., Asano K., Watari T.: Pulmonary adenosquamous carcinoma in a dog. J. Vet. Med. A Physiol. Pathol. Clin. Med. 2005, 52, 510-513, doi: 10.1111/j.1439-0442.2005.00773.x.

13. Sharp J. M., Angus K. W., Jassim F. A., Scott F. M.: Experimental transmission of sheep pulmonary adenomatosis to a goat. Vet. Rec. 1986, 119, 245.

14. Sharp J. M., DeMartini J. C.: Natural history of JSRV in sheep. Curr. Top. Microbiol. Immunol. 2003, 275, 55-79.

15. Sonawane G. G., Tripathi B. N., Kumar R., Kumar J.: Diagnosis and prevalence of ovine pulmonary adenocarcinoma in lung tissues of naturally infected farm sheep. Vet. World. 2016, 9, 365-370, doi: 10.14202/vetworld.2016.365-370.

16. Sousa Z. D., Rivera C. L., Quevedo C. D., Gorino A. C., Biagio C. S., Laufer A. R.: Pulmonary adenocarcinoma in cattle. Rev. MVZ Cordoba 2014, 19, 4358-4363 .

17. Travis W. D., Brambilla E., Nicholson A. G., Yatabe Y., Austin J. H. M., Beasley M. B.: The 2015 World Health Organization Classification of Lung Tumors: Impact of Genetic, Clinical and Radiologic Advances Since the 2004 Classification. J. Thorac. Oncol. 2015, 10, 1243-1260, doi: 10.1097/ JTO.0000000000000630.

18. Ujvari B., Roche B., Thomas F.: Ecology and Evolution of Cancer $-1^{\text {st }}$ Edition. Elsevier, London 2017.

19. Wandera J. G.: Sheep pulmonary adenomatosis (Jaagsiekte). Adv. Vet. Sci. Comp. Med. 1971, 15, 251-283.

20. Zhang K., Kong H., Liu Y., Shang Y., Wu B., Liu X.: Diagnosis and phylogenetic analysis of ovine pulmonary adenocarcinoma in China. Virus Genes. 2014, 48, 64-73, doi: 10.1007/s11262-013-0988-x.

Corresponding author: Michał Bednarski DVM, PhD, pl. Grunwaldzki 45, 50-366 Wrocław; e-mail: michal.bednarski@upwr.edu.pl 